${ }^{3}$ Metabolic Disease Research Center, Qazvin University of Medical Science, Qazvin, Iran

${ }^{4}$ Qazvin University of Medical Sciences, Qazvin, Iran

${ }^{5}$ School of Nursing and Midwifery, Mashhad University of Medical Sciences, Mashhad, Iran

\title{
The relationship between fear of hypoglycemia and sleep quality among type 2 diabetic patients
}

\section{ABSTRACT}

Background. Fear of hypoglycemia can result in anxiety, stress, anger, depression and severe avoidance behaviors that it affects the sleep quality of diabetic patients. Therefore, the present study was conducted with the aim of investigating the relationship between fear of hypoglycemia and sleep quality among type 2 diabetic patients.

Methods. The present cross-sectional study was conducted on 400 type 2 diabetic patients referred to endocrinology clinic of Velayat Hospital and Boali Hospital in Qazvin, in 2019. Data were collected using a checklist for demographic variables, the Fear of Hypoglycemia Survey (FHS-W), and the Pittsburgh sleep quality index (PSQI). Descriptive statistics and Spearman correlation test were performed for data analysis using SPSS v24. Results. In this study, the mean age of diabetic patients was $\mathbf{5 5 . 7 5} \pm \mathbf{1 0 . 3 1}$. The majority of the participants were female ( $n=299,74.8 \%$ ) and were treated with oral anti-diabetic drugs ( $n=174,43.5 \%$ ). The mean score of sleep quality in patients was $8.98 \pm 3.64$ and the fear of hypoglycemia was $21.27 \pm 11.92$. The results of this study showed that there was a significant

Address for correspondence:

Mohamad Hossein Mafi

School of Nursing and Midwifery

Mashhad University of Medical Sciences

Postal Code: 3419759811, Mashhad, Iran

Phone: +989125818173

Fax: +982832237268

e-mail: mafi1404@gmail.com

Clinical Diabetology 2020, 10, 1: 149-154

DOI: $10.5603 /$ DK.2020.0057

Received: 10.06.2020

Accepted: 02.11.2020 relationship between the fear of hypoglycemia and the poor sleep quality among patients $(P<0.001$, $r=0.305$ ).

Conclusion. The fear of hypoglycemia has a direct and significant relationship with poor sleep quality in diabetic patients; so that this fear reduces the quality of sleep in diabetic patients. Therefore, in order to provide adequate sleep to prevent inappropriate sleep complications, great attention should be paid to the issue of fear of hypoglycemia, and consider some actions to reduce this fear. (Clin Diabetol 2020; 10, 1: 149-154)

Key words: fear of hypoglycemia, sleep quality, type 2 diabetes

\section{Introduction}

Diabetes is not only recognized as a disease; but also, it is a collection of metabolic diseases that are caused by a disruption of the insulin's secretion or/and function, which is associated with an increase in the blood glucose level and a disruption of the metabolism of carbohydrates, lipids, and proteins [1]. According to the statistics, the number of diabetic people by the year 2035 is estimated to be 600 million. In Iran, studies have reported the prevalence of diabetes to be $9.6 \%$, accounting for almost 5 million patients. Also, it is estimated that the number of diabetic patients will be tripled every 15 years [2, 3]. In addition to widespread prevalence of this disease, the multiple and disabling complications of it is an important issue [4].

One of the most important complications of diabetes known to be a serious and clinical concern in patients with diabetes mellitus is hypoglycemia [5]. 
Therefore, one of the main goals of the treatment in these patients is the prevention of hypoglycemia. This matter makes it possible to achieve the ultimate goal in the treatment of diabetes, which is to control the level of blood glucose and prevent the complications of the disease [6]. Hypoglycemia can be very destructive in every aspect of everyday life, such as performance, work, personal relationships, and recreational activities, and may cause fear of hypoglycemia in individuals [7]. Severe and frequent hypoglycemia leads to fear of hypoglycemia [8].

Fear of hypoglycemia is associated with severe symptoms, such as anxiety, in diabetics and their families [9]. Patients with fear of hypoglycemia may keep their blood glucose levels high in order to prevent hypoglycemia [10], which it can affect long-term complications of diabetes; therefore, this fear results in poor control of hypoglycemia and complications of diabetes. This fear in many people, besides the physical consequences caused by different behaviors, leads to feeling of shame due to loss of control, irritation, confusion, and reduction in the quality of life $[5,11]$. Factors associated with fear of hypoglycemia include the variability in blood glucose level, the length of time since the first insulin treatment [12], and reduced awareness of hypoglycemia [13]. The fear of hypoglycemia may vary at different times and may be increased or decreased based on the perception of individuals or the risk of actual hypoglycemia [14]. Fear of hypoglycemia, unlike many phobias, is not only abnormal, but also a little fear of hypoglycemia, especially of severe one, is essential [15]; however, some patients develop panic attacks followed by this fear that it can lead to anxiety, stress, anger, depression and severe avoidance behaviors, and affect their sleep quality [16].

One of the most important factors in the life of diabetic patients is their sleep quality; because sleep disturbance has an adverse effect on their performance, as well as their physical and mental health. Sleep quality is a mental index related to sleep experiences, such as satisfaction with sleep and a sense of vitality from it [17]. Insomnia has a two-way relationship with the prevalence and incidence of diabetes, it can sometimes be secondary to diabetes or it can be a cause for diabetes itself. Recent studies have shown that increasing or decreasing the length of sleep at night is associated with the increased prevalence and incidence of diabetes, or inappropriate control of blood glucose level in diabetic patients [18]. In some studies, besides the effects of diabetes on sleep quality, the relationship between diabetes complications and patients' sleep quality has been studied. For instance, a study reported that the fear of hypoglycemia reduced the individuals' sleep quality. In this study, it was also stated that this disorder may be due to a behavioral disorder in sleep (for example, waking up during the night to check the blood glucose level) [16]. In another study, diabetic patients reported poor sleep quality after a nocturnal hypoglycemic event; so that $13.4 \%$ of them could not return to sleep at night, and only $32.4 \%$ reported that they experienced a good night of sleep [19]. On the other hand, Beléndez et al. (2009) reported that fear of hypoglycemia often occurs at night, which it can be one of the causes of insomnia [20].

In a study by Martyn-Nemeth et al. (2016), the evidence suggests that fear of hypoglycemia may contribute to poor sleep quality; therefore, further studies are needed to determine the association between the fear of hypoglycemia and sleep quality [21]. Given the limited information in this matter, especially among the Iranian society, the present study was conducted with the aim of investigating the relationship between the fear of hypoglycemia and sleep quality among type 2 diabetic patients.

\section{Materials and methods}

The present cross-sectional study was conducted on 400 type 2 diabetic patients referred to endocrinology clinic of Velayat Hospital and boali Hospital in Qazvin, in 2019. Patients were eligible to participate in the study if they aged 18 to 65 , were willing to participate in the study and diagnosed with type 2 diabetes by a physician or under the treatment of diabetes for at least one year. Pregnant women, type 1 diabetic patients, and those who had experienced acute psychiatric illnesses or special diseases during the past 6 weeks (according to a physician's diagnosis) were excluded. In this study, diabetic patients were randomly recruited through a convenience sampling method. Sample size was estimated to be 400 patients based on the previous study [22] and the sample size formula. After identifying the eligible participants, the questionnaires were distributed among them by researchers.

Data were collected using a checklist for demographic variables, the Fear of Hypoglycemia Survey, and the Pittsburgh sleep quality index (PSQI). The checklist for demographic variables of patients included information about their age, gender, marital status, education, duration of disease, type of treatment, having a history of complications and underlying illness.

The sub-scale of the Fear of Hypoglycemia Survey (FHS-W) consists of 18 items that was used to measure the patients' worries about hypoglycemia and its negative effects in the past six months and its score ranges from 0 to 72 (0 [never worry] to 4 [always worry]). Higher score indicates increased fear of hypoglyce- 
mia. This survey was used in a study by Momeni et al. (2016) and its alpha coefficient was determined to be 0.87 and the reliability was reported to be 0.76 using a test-retest method [22].

The Pittsburgh sleep quality index (PSQI) was used to measure the sleep quality of diabetic patients. This questionnaire was developed with the aim of measuring the sleep quality of patients over the past month and consists of 18 items. This questionnaire includes seven domains: subjective sleep quality, sleep latency, sleep duration, habitual sleep efficiency, sleep disturbances, use of sleep medication, and daytime dysfunction. Scoring of the answers in each domain is based on a 0 to 3 scale from 0 (no difficulty), to 3 (severe difficulty). The domains scores are summed to produce a global score that ranges from 0 to 21 . The higher the score is, the lower the sleep quality. A global sum of ' 5 ' or greater indicates a poor sleeper and the lower score reflects the patient's proper sleep quality [23]. The validity of the PSQI was determined to be 0.8 with the alpha coefficient and reported to be from 0.93 to 0.98 using a test-retest method [24].

Collected data were analysed using SPSS v24. Descriptive (mean, standard deviation, frequency and percentage frequency) and inferential (Spearman correlation test) statistics were performed for data analysis.

\section{Results}

In this study, the mean age of patients was 55.75 \pm 10.31 . The majority of the participants were female $(n=299,74.8 \%)$ and were married $(n=388,97.2 \%)$.

In terms of clinical findings, 174 (43.5\%) of the diabetic patients used oral drugs to control their diabetes, and most of them had complications (89.3\%) and Concomitant (88.8\%). The most common complication of diabetes in this study was nervous complication (59.4\%). Most patients had a combination of diseases as a concomitant disease (63.7\%) (Table $1)$. In this study, the mean score of the fear of hypoglycemia in patients was $21.27 \pm 11.92$ and the sleep quality was $8.98 \pm 3.64$. The score of the different domains of sleep quality were also calculated and reported in Table 2 .

The relationship between the fear of hypoglycemia and sleep quality among diabetic patients was investigated and based on it, a significant relationship was observed between the fear of hypoglycemia and the sleep quality among diabetic patients $(P<0.001$, $r=0.305$ ) (Figure 1). Based on scores, higher scores of sleep quality indicate poor sleep quality in diabetic patients (Table 3).

Also, the relationship between the fear of hypoglycemia and the domains of sleep quality was inves-
Table 1. Patients' demographic variables

\begin{tabular}{|c|c|}
\hline Age & $55.75 \pm 10.31$ \\
\hline Duration of disease & $10.45 \pm 7.11$ \\
\hline \multicolumn{2}{|l|}{ Gender } \\
\hline Female & $299(74.8 \%)$ \\
\hline Male & $101(25.2 \%)$ \\
\hline \multicolumn{2}{|l|}{ Marital status } \\
\hline Single & $11(2.8 \%)$ \\
\hline Married & $388(97.2 \%)$ \\
\hline \multicolumn{2}{|l|}{ Education } \\
\hline Illiterate & $194(48.5 \%)$ \\
\hline Under diploma & $162(40.5 \%)$ \\
\hline Diploma & $35(8.7 \%)$ \\
\hline University degree & $9(2.3 \%)$ \\
\hline \multicolumn{2}{|l|}{ Type of treatment } \\
\hline Insulin & $124(31.0 \%)$ \\
\hline Oral drugs & $174(43.5 \%)$ \\
\hline Combinatory & $102(25.5 \%)$ \\
\hline \multicolumn{2}{|l|}{ Complications } \\
\hline Yes & $357(89.3 \%)$ \\
\hline No & $43(10.7 \%)$ \\
\hline \multicolumn{2}{|l|}{ Type of complications } \\
\hline Renal & $8(2.2 \%)$ \\
\hline Cardiovascular & $6(1.7 \%)$ \\
\hline Ocular & $18(5 \%)$ \\
\hline Nervous & $212(59.4 \%)$ \\
\hline Combination of complications & $113(31.7 \%)$ \\
\hline \multicolumn{2}{|l|}{ Concomitant } \\
\hline Yes & $355(88.8 \%)$ \\
\hline No & $45(11.2 \%)$ \\
\hline \multicolumn{2}{|l|}{ Type of concomitant disease } \\
\hline Cardiovascular & $60(16.9 \%)$ \\
\hline Kidney disease & $9(2.5 \%)$ \\
\hline Thyroid disease & $13(3.7 \%)$ \\
\hline Hyperlipidemia & $36(10.1 \%)$ \\
\hline Other diseases & $11(3.1 \%)$ \\
\hline Combination of diseases & $226(63.7 \%)$ \\
\hline
\end{tabular}

Table 2. Patients' overall score of fear of hypoglycemia and sleep quality

\begin{tabular}{lc}
\hline Overall score of fear of hypoglycemia & $21.27 \pm 11.92$ \\
Overall score of sleep quality & $8.98 \pm 3.64$ \\
Domains of sleep quality & \\
Subjective sleep quality & $1.42 \pm 0.85$ \\
Sleep latency & $1.82 \pm 0.95$ \\
Sleep duration & $0.88 \pm 0.99$ \\
Habitual sleep efficiency & $0.90 \pm 1.00$ \\
Sleep disturbances & $1.89 \pm 0.56$ \\
Use of sleep medication & $0.74 \pm 1.02$ \\
Daytime dysfunction & $1.27 \pm 0.79$ \\
\hline
\end{tabular}




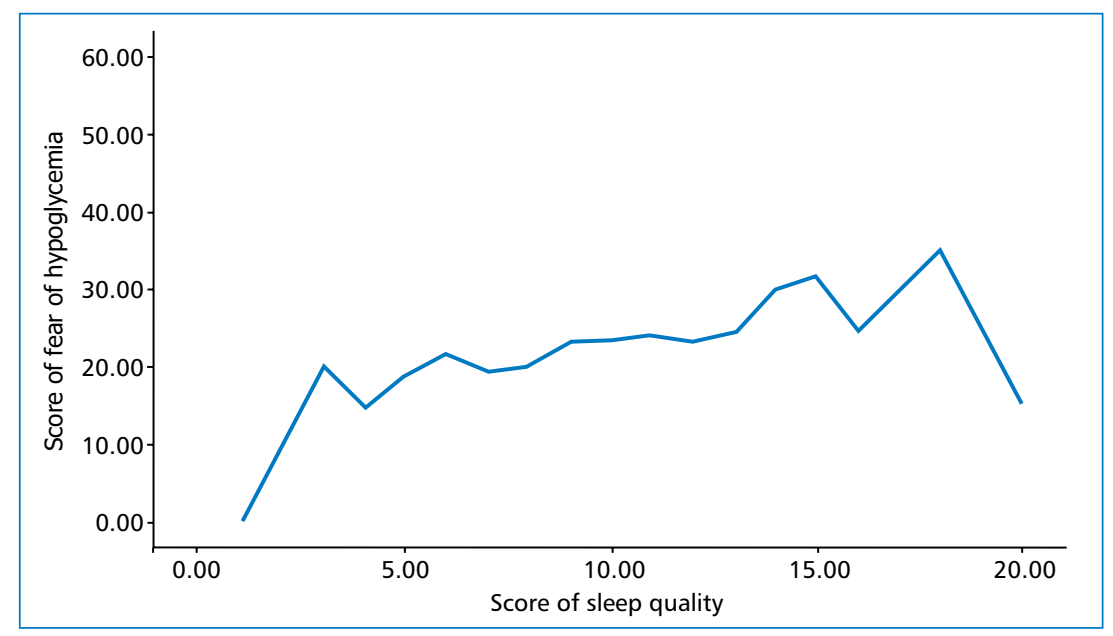

Figure 1. The relationship between the fear of hypoglycemia and sleep quality

Table 3. The relationship between the fear of hypoglycemia and sleep quality

\begin{tabular}{lcc}
\hline & \multicolumn{2}{c}{ Fear of hypoglycemia } \\
\cline { 2 - 3 } & $\mathbf{r}$ & $\mathbf{P}$ \\
\hline Subjective sleep quality & 0.251 & $<0.001$ \\
Sleep latency & 0.181 & $<0.001$ \\
Sleep duration & 0.039 & 0.445 \\
Habitual sleep efficiency & 0.074 & 0.150 \\
Sleep disturbances & 0.320 & $<0.001$ \\
Use of sleep medication & 0.130 & 0.012 \\
Daytime dysfunction & 0.388 & $<0.001$ \\
Overall score of sleep quality & 0.305 & $<0.001$ \\
\hline
\end{tabular}

tigated. According to the results, hypoglycemia had a significant relationship with domains such as subjective sleep quality $(P<0.001)$, sleep latency $(P<0.001)$, sleep disturbances $(P<0.001)$, use of sleep medication $(P=0.012)$, daytime dysfunction $(P<0.001)$ (Table 3$)$.

\section{Discussion}

Diabetes is one of the most common metabolic diseases worldwide that is associated with many complications. These complications can affect different dimensions of the patients' life and sleep, and reduce the quality of life and sleep quality of them.

The main goals in treatment of diabetic patients are to improve the sleep quality and control the side effects of the disease, which it will improve the quality of life of these individuals so as to experience a normal life. In order to achieve this goal, it is necessary to diagnose the complications of diabetes and their effect on the sleep quality of diabetic patients.
According to the results, the overall score of patients' sleep quality was $8.98 \pm 3.64$. Based on the scoring of the questionnaire, a score of ' 5 ' or greater indicates a poor sleep quality among diabetic patients. The results of this study were in consistent with the results of Sadeghi Sedeh et al. (2017) study [25]. In the Jin et al. study, type 2 diabetic patients had poor sleep quality. Researchers have shown that sleep quality probably affects blood glucose regulation, and is closely correlated with the occurrence of complications. In addition, poor sleep quality results in poor life quality [26]. In another study, sleep disturbances in diabetic patients were reported more often than others, and according to the findings, these disorders have negative implications for glycemic control and diabetes management, as well as psychosocial and cognitive outcomes [27].

In this study, the fear hypoglycemia among diabetic patients was studied as well. The score in the Fear of Hypoglycemia Survey (FHS-W) ranges from 0 to 72 and higher score indicates increased fear of hypoglycemia. The mean score of the fear of hypoglycemia in patients was $21.27 \pm 11.92$. In the study of Mahabalshetti et al. (2016), the score of the fear of hypoglycemia among patients with uncontrolled diabetes was $35.07 \pm 8.38$. Perhaps this difference can be due to the difference in the study population; because the complications of diabetes in people with uncontrolled diabetes are more. On the other hand, the questionnaire used was different from the questionnaire of the present study [28]. In a study by Momeni et al. (2016), the score of the fear of hypoglycemia among diabetic patients who only used oral drugs to control diabetes was $16.8 \pm$ 16.33 [22]. The differences in the score of the fear of hypoglycemia among type 2 diabetic patients in two studies, based on other studies, such as Beléndez et al. 
(2009) [20] and Erol et al. (2011) [8], can be attributed to the use of different treatments for controlling diabetes; In the study of Momeni et al. (2016), the fear of hypoglycemia was measured only in patients who used oral drugs. However, in the present study, diabetic patients used different types of treatments (insulin, oral drug and. combination of insulin and oral drug) [22].

The main objective of this study was to determine the relationship between the fear of hypoglycemia and sleep quality among type 2 diabetic patients. The results of the correlation test in this study showed a direct and significant relationship between the fear of hypoglycemia and sleep quality among diabetic patients, so that the score of sleep quality of patients increased significantly due to the increased score of the fear of hypoglycemia. Since the increased score of the fear of hypoglycemia indicated poor sleep quality among the patients, fear of hypoglycemia reduced the sleep quality of type 2 diabetic patients. The results of this study are confirmed by other studies including Martyn-Nemeth et al. (2014) and Brod et al. (2012) studies [16, 19]. In another study, the results indicated that nearly half of the participants were worried about insulin reactions during sleep. For this reason, patients were more afraid of hypoglycemia during the night, and it affected the sleep quality of them and sometimes led to obsessive behaviors, such as frequently checking the blood glucose level overnight [21].

\section{Conclusion}

Based on the results of the present study, there was a direct and significant relationship between the fear of hypoglycemia and the poor sleep quality among type 2 diabetic patients. This issue itself has negative implications for diabetes control and diabetes management, as well as with psychosocial and cognitive outcomes that ultimately reduce the quality of life of the diabetic disease. Given the few studies on the fear of hypoglycemia and its impact on the components of patients' quality of life, such as sleep quality, in Iranian society, it can be concluded that this matter is a neglected topic in the field of research and care. So, the researchers of the present study studied this issue and the findings indicated a significant relationship between these two variables. As a result, studying the fear of hypoglycemia is considered necessary in preventing other complications of diabetes and complications associated with the fear of hypoglycemia among diabetic patients. On the other hand, it may be possible to provide the necessary training to control this fear and consequently eliminate the factor affecting the patients' sleep quality and improve it among diabetic patients.

\section{Suggestions}

Given the prevalence of complications such as reduced sleep quality, and the fear of hypoglycemia among diabetic patients, it is recommended that researchers provide an educational program in order to control the complications of diabetes and improve the patients' quality of life. It is also recommended that further studies be conducted to determine the effect of self-care educational program on the sleep quality as well as the fear of hypoglycemia.

\section{Limitation}

Considering only one type of diabetes, one clinic and the cross-sectional design in this study can be addressed as the limitation of the present study. The strengths of this study were the great number of patients and collecting data through interviewing with diabetic patients.

\section{Acknowledgement}

This article is the result of a proposal approved by Qazvin University of Medical Sciences as well as the Ethics Committee of the University (ethics code: IR.QUMS.REC.1398.080). The authors would like to thank all the patients, authorities of the Velayat Hospital and the professors of the Qazvin school of nursing and midwifery for their cooperation to conduct this study.

\section{Conflict of interest}

The authors declare to have no conflict of interest.

\section{REFERENCES}

1. Olfatifar M, Karami M, Shokri P, et al. Prevalence of chronic complications and related risk factors of diabetes in patients referred to the Diabetes Center of Hamedan Province. Scientific Journal of Hamedan Nursing and Midwifery Faculty. 2017; 25(2): 69-74, doi: 10.21859/nmj-25029.

2. Bakker K, Apelqvist J, Lipsky BA, et al. International Working Group on the Diabetic Foot. The 2015 IWGDF guidance documents on prevention and management of foot problems in diabetes: development of an evidence-based global consensus. Diabetes Metab Res Rev. 2016; 32 Suppl 1: 2-6, doi: 10.1002/dmrr.2694, indexed in Pubmed: 26409930.

3. Khandouzi N, Shidfar $F$, Rajab A, et al. The effects of ginger on fasting blood sugar, hemoglobin a1 C, apolipoprotein B, apolipoprotein a-I and malondialdehyde in type 2 diabetic patients. Iran J Pharm Res. 2015; 14(1): 131-140, indexed in Pubmed: 25561919.

4. YekeFallah L, Talebi F, Ghorbani A, Mafi M, Mafi MH. The relationship between fear of hypoglycemia and quality of life in patients with type 2 diabetes. Iranian Journal of Endocrinology and Metabolism. 2019; 21(3): 138-144.

5. Hazavehei SM, Khani Jeihooni A, Hasanzadeh A, et al. The effect of educational program based on BASNEF model for eye care in non-insulin dependent diabetic patients. J Res Health Sci. 2010; 10(2): 81-90, indexed in Pubmed: 22911929.

6. Williams SA, Pollack MF, Dibonaventura M. Effects of hypoglycemia on health-related quality of life, treatment satisfaction and healthcare resource utilization in patients with type 2 diabetes 
mellitus. Diabetes Res Clin Pract. 2011; 91(3): 363-370, doi: 10.1016/j.diabres.2010.12.027, indexed in Pubmed: 21251725.

7. Zammitt NN, Frier BM. Hypoglycemia in type 2 diabetes: pathophysiology, frequency, and effects of different treatment modalities. Diabetes Care. 2005; 28(12): 2948-2961, doi: 10.2337/ diacare.28.12.2948, indexed in Pubmed: 16306561.

8. Erol O, Enc N. Hypoglycemia fear and self-efficacy of Turkish patients receiving insulin therapy. Asian Nurs Res (Korean Soc Nurs Sci). 2011; 5(4): 222-228, doi: 10.1016/j.anr.2011.12.001, indexed in Pubmed: 25030524.

9. Gonder-Frederick L, Nyer M, Shepard JA, et al. Assessing fear of hypoglycemia in children with Type 1 diabetes and their parents. Diabetes Manag (Lond). 2011; 1(6): 627-639, doi: 10.2217/ DMT.11.60, indexed in Pubmed: 22180760.

10. Green LB, Wysocki T, Reineck BM. Fear of hypoglycemia in children and adolescents with diabetes. J Pediatr Psychol. 1990; 15(5): 633-641, doi: 10.1093/jpepsy/15.5.633, indexed in Pubmed: 2283572.

11. Snoek FJ, Kersch NYA, Eldrup E, et al. Monitoring of individual needs in diabetes (MIND): baseline data from the cross-national diabetes attitudes, wishes, and needs (DAWN) MIND study. Diabetes Care. 2011; 34(3): 601-603, doi: 10.2337/dc10-1552, indexed in Pubmed: 21266654.

12. Wild D, von Maltzahn R, Brohan E, et al. A critical review of the literature on fear of hypoglycemia in diabetes: Implications for diabetes management and patient education. Patient Educ Couns. 2007; 68(1): 10-15, doi: 10.1016/j.pec.2007.05.003, indexed in Pubmed: 17582726

13. Hepburn DA, Deary IJ, MacLeod KM, et al. Structural equation modeling of symptoms, awareness and fear of hypoglycemia, and personality in patients with insulin-treated diabetes. Diabetes Care. 1994; 17(11): 1273-1280, doi: 10.2337/diacare.17.11.1273, indexed in Pubmed: 7821167.

14. Cox DJ, Gonder-Frederick L, Antoun B, et al. Psychobehavioral metabolic parameters of severe hypoglycemic episodes. Diabetes Care. 1990; 13(4): 458-459, doi: 10.2337/diacare.13.4.458, indexed in Pubmed: 2318112.

15. Anderbro T, Amsberg S, Adamson U, et al. Fear of hypoglycaemia in adults with Type 1 diabetes. Diabet Med. 2010; 27(10): 1151-1158, doi: 10.1111/j.1464-5491.2010.03078.x, indexed in Pubmed: 20854383.

16. Martyn-Nemeth $P$, Quinn L. Poor sleep quality is associated with depressive mood, low self-esteem, and fear of hypoglycemia among young adults with type 1 diabetes. InDiabetes. 2014; 63(1): 208.

17. Leila Dehghankar L, Ghorbani A, Yekefallah L, Hajkarimbaba M, RostampourA. Association of sleep quality with socio-demo- graphic characteristics in elderly Referred to health centers in Qazvin, Iran. Sleep Hypn. 2018; 20(3): 227-232. http://dx.doi. org/10.5350/Sleep.Hypn.2017.19.0154.

18. Maracy MR, Kheirabadi GR, Fakhari N, et al. Comparison of night time sleep quality in type 2 diabetics, impaired glucose tolerance cases and non-diabetics. IJEM. 2011; 13(2): 165-72.

19. Brod M, Christensen T, Bushnell DM. Impact of nocturnal hypoglycemic events on diabetes management, sleep quality, and next-day function: results from a four-country survey. J Med Econ. 2012; 15(1): 77-86, doi: 10.3111/13696998.2011.624144, indexed in Pubmed: 22029460.

20. Beléndez $M$, Hernández-Mijares $A$. Beliefs about insulin as a predictor of fear of hypoglycaemia. Chronic IIIn. 2009; 5(4): 250-256, doi: 10.1177/1742395309346464, indexed in Pubmed: 19933244.

21. Martyn-Nemeth P, Schwarz Farabi S, Mihailescu D, et al. Fear of hypoglycemia in adults with type 1 diabetes: impact of therapeutic advances and strategies for prevention - a review. J Diabetes Complications. 2016; 30(1): 167-177, doi: 10.1016/j. jdiacomp.2015.09.003, indexed in Pubmed: 26439754.

22. Momeni M, Ziaee A, Ghorbani A. Predictors of hypoglycemia fear in patients with type 2 diabetes under treatment of oral anti hyperglycemic agents. IJEM. 2016; 18: 28-36.

23. Agargun MY, Kara H, Anlar Ö. Validity and reliability of the Pittsburgh Sleep Quality Index in Turkish sample. Turk J Psychiat . 1996; 7: 107-115, doi: 10.5606/ArchRheumatol.2018.6376, indexed in Pubmed: 30207571.

24. Yousefi F, Sharifi Z. Investigation of sleep quality and its influencing factors in patients admitted to the gynecology and general surgery of Besat Hospital in Sanandaj. MJMS. 2014; 57(6): 762-769.

25. Sadeghi Sedeh B, Talaei A, Parham M. Comparison of quality and type of sleep disorders in good control and uncontroled diabetic type2 patients. J Shahrekord Univ Med Sci. 2017; 19(3): 65-75.

26. Jin $\mathrm{QH}, \mathrm{Chen} \mathrm{HH}, \mathrm{Yu} \mathrm{HL}$, et al. [The relationship between sleep quality and glucose level, diabetic complications in elderly type 2 diabetes mellitus]. Zhonghua Nei Ke Za Zhi. 2012; 51(5): 357-361, indexed in Pubmed: 22883333.

27. Perez KM, Hamburger ER, Lyttle M, et al. Sleep in type 1 diabetes: implications for glycemic control and diabetes management. Curr Diab Rep. 2018; 18(2): 5, doi: 10.1007/s11892-018-0974-8, indexed in Pubmed: 29399719.

28. Mahabalshetti AD, Ramdurg S, Dhananjaya M. Prevalence of fear of hypoglycemic attack in patients with uncontrolled diabetes mellitus and correlation analysis in diabetes. IJSS. 2016; 3: 169-173. 